\title{
La poesía en el aula de español como lengua extranjera. Explotación didáctica de un poema de Juana Castro para el nivel avanzado de EEOOI'
}

Poetry in the Classroom of Spanish as a Foreign Language. Didactic Exploitation of a Poem By Juana Castro for the Advanced Level Of Official Language Schools in Spain

\author{
RAQUEL LANSEROS SANCHEZ \\ University of West Florida \\ Estados Unidos \\ raqlanseros@yahoo.es
}

(Recibido: 23-O4-20I7; aceptado: 2O-O8-20I7)

Resumen. La poesía es una de las herramientas más útiles con las que cuenta el profesor en el proceso de enseñanzaaprendizaje de la lengua. Este trabajo presenta una propuesta didáctica dirigida al nivel avanzado de español como lengua extranjera de las Escuelas Oficiales de Idiomas, correspondiente al nivel B2 del Marco Común Europeo de Referencia para las Lenguas. Se pretende que el alumnado desarrolle su competencia lectora, su capacidad de análisis literario y su conocimiento de la cultura española, a la vez que se familiarizan con la poesía contemporánea. Partiendo de un poema de Juana Castro, se presentan una serie de actividades de profundización lingüística y de comprensión lectora, así como iniciativas de fomento de la lectura. Se proporciona asimismo a los docentes una serie de herramientas básicas de evaluación, con las cuales podrán definir el progreso de los alumnos y su comprensión e interpretación del texto poético.

Palabras clave: poesía; E/LE; Juana Castro; educación literaria; nivel avanzado de las Escuelas Oficiales de Idiomas.
Abstract. Poetry is one of the most useful tools for the teacher in the language teaching-learning process. This paper presents a didactic proposal directed at the advanced level of Spanish as a foreign language of the Official Language Schools, corresponding to level B2 of the Common European Framework of Reference for Languages. It is intended to help students develop their reading and literary analysis skills, as well as knowledge of Spanish culture, while becoming familiar with contemporary poetry. Starting with a poem by Juana Castro, we present a series of activities to deepen linguistic and reading comprehension, as well as some initiatives to promote reading. Teachers are provided with a series of basic assessment tools, so that they can evaluate students' progress and their understanding and interpretation of poetic texts.

Keywords: poetry; spanish as a foreign language; Juana Castro; literary Education; Advanced Level of the Official Language Schools.

\footnotetext{
I Para citar este artículo: Lanseros Sanchez, Raquel (20I8). La poesía en el aula de español como lengua extranjera. Exploración didáctica de un poema de Juana Castro para el nivel avanzado de EEOOII. Alabe 17. [www.revistaalabe.com]

DOI: IO.I5645/Alabe20I8.I7.4
} 


\section{Introducción}

El conocimiento de la cultura que rodea a la lengua extranjera que desea adquirirse, y más concretamente de su literatura, ayuda a desarrollar una valoración más positiva de ella, además de hacer al estudiante partícipe de conocimientos que favorecen su proceso de aprendizaje. Este refuerzo de la ligazón afectiva del alumno hacia la lengua que estudia se produce de una forma decidida mediante el uso y análisis de la poesía en el aula, puesta que ésta potencia inferencias estéticas de índole universal que facilitan el funcionamiento de la memoria y la individualización del vocabulario y las estructuras lingüísticas.

Además, la exposición a los textos poéticos genera una respuesta emocional muy favorecedora del proceso de aprendizaje, alejándolo de las propuestas gramaticalistas conservadoras, más frías y rutinarias. Es innegable además, como apunta Albaladejo "la necesidad de una revalorización de su potencial como fuente de inspiración para la creación de actividades comunicativas, a la vez que integradoras de las cuatro destrezas lingüísticas" (2007:2).

Es muy importante que la presentación se realice utilizando una metodología activa y dinámica, alejada del modelo lingüístico y normativo para los estudiantes. Siendo así, se trata de un indispensable vehículo de conocimiento cultural y un motivador instrumento de trabajo, en virtud del atractivo que posee para el estudiante.

Para poder sacar partido de estos objetivos, primero hemos de vincular el uso de la poesía en el aula de español como lengua extranjera con los intereses del alumnado. Tenemos que tener presente que el nivel de aplicación más adecuado es el avanzado, correspondiente al nivel B2 del Marco Común Europeo de Referencia para las Lenguas. Para el presente trabajo de aplicación didáctica de la poesía de Juana Castro al nivel avanzado del aula de español como lengua extranjera, hemos elegido un poema, con el cual hemos construido una propuesta didáctica no evaluable en términos convencionales, que constaría de tres a cuatro horas académicas, dependiendo del ritmo de aprendizaje de los estudiantes.

Creemos que la presentación de formas más elaboradas y artísticas de la lengua española, como son los poemas, servirá de instrumento de conocimiento amplio de la cultura, así como de reflexión lingüística. Algunos de estos objetivos que alcanzaremos con la introducción de la poesía en el aula de español como lengua extranjera son los siguientes:

- Fomentar la capacidad de distinción entre los significados literales y simbólicos o metafóricos.

- Proporcionar una visión más profunda de la compleja realidad que supone toda lengua.

- Motivar al alumnado mediante la exposición de textos de gran contenido artístico.

- Ampliar el corpus de vocabulario utilizado, preferentemente en los cursos avanzados.

- Profundizar en el conocimiento de la cultura propia de los países de habla hispana.

- Despertar el interés por la poesía en español más allá de nuestras fronteras. 


\section{Justificación de la propuesta didáctica}

Hemos seleccionado un poema de la poeta Juana Castro para efectuar nuestra propuesta didáctica de desarrollo y puesta en práctica en el aula. Se trata de familiarizar al alumnado con el lenguaje poético y, más allá, con el universo literario de Juana Castro, quien ha sido capaz de aunar en su producción la esencialidad y la búsqueda de la verdad, configurando un territorio en el que las tierras y las lenguas se funden en un espacio común de conocimiento.

Intentaremos abundar en la identificación del alumnado con los pensamientos y sentimientos de Juana Castro expresados en su poesía, como medio para comprender la falta de barreras temporales, generacionales, espaciales o lingüísticas en la poesía. Como la propia autora expresa, refiriéndose a la importancia del uso didáctico de la poesía en el aula (Lanseros, 20I6: 193):

No existe persona que no pueda ser tocada por el misterio y la indecibilidad de la poesía. Pero para conseguirlo hay que crear el clima adecuado y hacer lecturas individuales y corales, hasta que cada alumna y cada alumno paladee las palabras, las vea y las sienta como carne de su carne.

Para la selección de este poema, hemos tenido en cuenta el especial significado simbólico dentro de su trayectoria vital y poética. En el poema “Toda la piel del mundo", Juana Castro nos habla -con una mezcla de ironía y ternura- del bolso de las mujeres, inseparable compañero urbano y complemento indispensable, que se transforma en el poema en un símbolo del altruismo y la dadivosidad histórica de las mujeres, que han venido siendo referentes de apoyo y ayuda, casi en cualquier situación, para sus familias. Del mismo modo, es una reivindicación de la condición femenina, de la mujer que afirma su identidad apoyándose en su propia fortaleza.

\section{Nivel de aplicación}

La poesía aúna, dentro de los géneros literarios, todas las funciones comunicativas que explicitara Roman Jakobson (I963). Su inclusión en el aula de español para extranjeros de las EEOOII es imprescindible para dotar a los alumnos de una formación lingüística que incluya contenidos socioculturales y desarrollo de la educación emocional en la lengua de aprendizaje. El propio Marco común europeo de referencia para las lenguas (MCER) lo explicita: "Los estudios literarios cumplen muchos más fines educativos, intelectuales, morales, emocionales, lingüísticos y culturales que los puramente estéticos" $(2002,60)$.

Conscientes de que su comprensión requiere unos conocimientos previos amplios de la lengua española, hemos situado nuestra propuesta didáctica en el nivel avanzado de la Enseñanza de Idiomas de Régimen Especial, del idioma español para extranjeros, nivel 
$\mathrm{B}_{2}$. Se trata del nivel más alto, dentro de la enseñanza de idiomas de régimen especial, y es, por tanto, el nivel más adecuado para la explotación didáctica del poema propuesto. La presente propuesta didáctica puede ser implementada en el primer o segundo curso del nivel avanzado, dependiendo del criterio personal del profesor y el grado global de conocimiento de cada grupo determinado.

Según el REAL DECRETO i629/2006, de 29 de diciembre, por el que se fijan los aspectos básicos del currículo de las enseñanzas de idiomas de régimen especial reguladas por la Ley Orgánica 2/2006, de 3 de mayo, de Educación; la definición del nivel avanzado es la siguiente: "El Nivel avanzado presentará las características del nivel de competencia B2, según este nivel se define en el Marco Común Europeo de Referencia para las Lenguas" (MCER).

El nivel avanzado tiene como finalidad capacitar a los alumnos para utilizar el idioma con soltura y eficacia en situaciones habituales y más específicas que requieran comprender, producir y tratar textos orales y escritos conceptual y lingüísticamente complejos, en una variedad de lengua estándar, con un repertorio léxico amplio aunque no muy idiomático, y que versen sobre temas generales, actuales o propios del campo de especialización del hablante.

\section{Aclaraciones metodológicas}

La metodología empleada tendrá un enfoque comunicativo y velará por la potenciación y el desarrollo de las cuatro destrezas comunicativas: comprensión oral, comprensión escrita, expresión e interactuación oral y expresión e interactuación escrita. Se tratará en todo momento de estimular una comprensión profunda del texto a través de la lectura, como afirman (López Valero y Encabo Fernández, 2OI3, 32-33):

El objetivo prioritario de la educación literaria deberá partir de la creación y fomento de su propia intertextualidad como elemento básico de su competencia literaria, que le permitirá establecer asociaciones entre la obra literaria percibida y otras manifestaciones artísticas, a partir de la actualización de sus propios conocimientos y del uso de estrategias y recursos múltiples, de manera que la relación establecida entre ése y otros muchos textos le sirva para construir de forma significativa su propio conocimiento y desarrollar su espíritu crítico.

La aproximación a la didáctica de la poesía ha de ser interactiva e incluir los siguientes elementos:

- Enfoque comunicativo.

- Clases prácticas, ágiles e innovadoras.

- Interpretación conjunta de los textos.

- Interacción continua profesor-alumno. 
- Aprendizaje cooperativo.

- Trabajo escrito e iniciación a la escritura creativa.

- Debate abierto sobre los múltiples sentidos y significaciones de la poesía.

- Análisis de los elementos culturales presentes.

- Uso de las TIC (plataformas, páginas web, blogs, webquests, etc.) para recabar y ampliar información.

En cuanto a la aplicación de la poesía al aula, es importante que el profesor no actúe tan sólo como transmisor de información, sino que se convierta en investigador de la propia acción educativa que está poniendo en práctica. De este modo, pasará a ser mediador entre su propia teoría y la praxis educativa implementada. Es fundamental resaltar que la lectura de textos literarios es una actividad abierta, en la cual el profesor se involucra en un proceso de negociación con los alumnos, a medida que diferentes percepciones e interpretaciones vayan aflorando. No existe una única manera de leer ni una única descodificación posible del texto leído, como ya advirtió Campos Fernández-Figares (2004: 46):

Cuando aprendimos a leer creíamos que las cosas eran de una sola manera. Eran así, tal y como nos las enseñaban. Y aprendíamos más: siempre habían sido así y por lógica en un futuro deberíamos esperar que siguieran de la misma manera. De todo esto se podrían extraer muchísimas consecuencias, evidentemente muchísimas contradicciones.

\section{Propuesta didáctica a partir de "Toda la piel del mundo", un poema de Cartas de enero, de Juana Castro}

\section{I. Planteamiento inicial}

El poema pertenece a Cartas de enero, publicado en 2010 en Sevilla por la Fundación José Manuel Lara, dentro del libro cuyo título global es Heredad seguido de Cartas de enero. Es importante realizar como paso inicial una contextualización históricoliteraria de la poeta cuyo poema seleccionado vamos a ir trabajando. Por ello, días antes de comenzar la actividad, deberemos proporcionar a los alumnos algunos enlaces donde buscar información sobre la poeta y su obra. Asimismo, se recomendará a los alumnos visitar la biblioteca pública más cercana a su domicilio, para buscar información sobre Juana Castro en libros de consulta, manuales de referencia o enciclopedias.

Para el día del comienzo de la actividad didáctica con el poema "Toda la piel del mundo", les habremos pedido que traigan redactada una pequeña biografía de la autora, en la que incluyan lo más representativo de su vida y su obra.

Comenzaremos la actividad leyendo en grupo unas cuantas biografías de la poeta escritas por los propios alumnos. El profesor aclarará aquellos detalles o aspectos tanto de su vida como de su obra poética que hayan podido pasar desapercibidos o no hayan 
sido comprendidos correctamente.

Una vez familiarizados con la figura del autor, procedemos a la lectura primero individual y posteriormente grupal de su poema "Toda la piel del mundo".

\section{TODA LA PIEL DEL MUNDO}

Tú los ves ahí colgados, tirados, y dices, vaya cosa, son cosa de mujeres, tonterías, lo llevan para meter el pintalabios, el móvil, quizás una compresa. Y te olvidas. Pero ellas no olvidan, lo llevan como a un gato, como al fiel compañero, como su santo y seña, como su claro ex-libris.

Te equivocas si crees, en tu inocencia, que esa cosa de rafia o de piel beige sirve para tener a mano el colorete, las llaves, el perfume.

Yo la he visto de noche, esa cosa respira, es una megalópolis, no está quieta por dentro, es multiforme y crece.

A la hora del pan huele a cerveza, y cuando está nublado te puedes encontrar con que ahí dentro hay una hija, un sol, unas tijeras de robar rosas rojas.

Ahí, a tres de julio, he visto amanecer los pájaros cantando y había un abanico para un novio y una estrella de miel para la madre. En el rincón azul, las gafas de coser, las recetas del padre a la fecha de hoy, la muestra de la tela -preciosa- que le dio el tapicero.

$\mathrm{Al}$ fondo la novela, la última, de Doris Lessing y el bono de ro horas del gimnasio.

Por ahí pasa un río, pasa el día, la música, la niebla... Esa cosa. Mi bolso.

Que va a dar al mar. 


\subsection{Actividades}

Los alumnos comenzarán leyendo en voz baja el poema “Toda la piel del mundo”, fijándose especialmente en las palabras, las construcciones gramaticales, sus imágenes y las sensaciones que los versos les provocan. Es preciso recordar que en las EEOOII los alumnos suelen ser muy heterogéneos en cuanto a su edad y bagaje cultural. El profesorado puede encontrarse trabajando el poema con adultos ya formados, pero también con adolescentes. Es muy importante que el docente adapte las actividades a la edad y experiencia cultural del alumnado concreto de cada curso. De este modo, le será más fácil crear un vínculo entre los intereses personales del alumno y la poesía en el aula. Por tanto, la actividad tendrá más éxito.

El libro al que pertenece el poema "Toda la piel del mundo" se titula Cartas de enero. Se interrogará a los alumnos sobre el posible significado de este título y se les pedirá que apunten sus ideas sobre él. A continuación, se pedirá a los alumnos que escriban una redacción breve expresando su propia interpretación del título Cartas de enero y sus estimaciones personales sobre el posible tema del libro.

Después, se pedirá a los alumnos que busquen en el diccionario las palabras que no comprendan. Una vez cerrados los diccionarios, y buscadas todas las palabras desconocidas, tratarán de escribir su propia definición de las siguientes palabras: Pintalabios, compresa, seña, exlibris, rafia, colorete, megalópolis, multiforme, abanico, coser, tapicero.

Seguidamente, reescribirán los siguientes versos tratando de encontrar un sinónimo para cada una de las palabras subrayadas:
Yo la he visto de noche,
esa cosa respira, es una megalópolis,
no está quieta por dentro, es multiforme y crece.

A continuación, reflexionarán sobre el resultado del ejercicio anterior, para intentar clarificar qué ha ocurrido al haber sustituido las palabras subrayadas por otras sinónimas. Reflexionarán sobre si ha cambiado mucho o no la estrofa y el poema. Asimismo, dilucidarán si los cambios han sido mayores en el significado o en la estética del poema (ritmo, musicalidad, belleza, etc.)

En el poema se enumeran muchos posibles objetos que pueden ir alojados en un bolso femenino. Se pedirá a los alumnos que los enumeren todos en una lista. Seguidamente, harán otra lista con objetos no mencionados en el poema, pero que también son frecuentemente transportados en un bolso. Finalmente, escribirán una redacción de aproximadamente ciento cincuenta palabras describiendo su propio bolso o bien el de una amiga o familiar. Se les pedirá que traten de detallar la materia de la que está compuesto, el color, el estilo y el contenido.

La siguiente actividad les propondrá intentar imaginar a la dueña del bolso de “Toda la piel del mundo". Por grupos, debatiremos sobre su posible edad, profesión o 
aficiones, basándonos en la información objetiva y subjetiva que nos proporciona el poema. Argumentaremos nuestras respuestas apoyándonos tanto en la información recibida como en nuestras sensaciones.

A continuación, trataremos de pensar en el título del poema, "Toda la piel del mundo" y en el significado que, en nuestra opinión tiene. Reflexionaremos sobre esa piel, tratando de adivinar qué metaforiza y a qué se puede estar refiriendo la poeta.

La palabra “piel” es polisémica, pues puede referirse tanto a la piel humana, como al cuero animal, como al propio contacto dérmico. Pediremos a los alumnos que piensen en dos palabras polisémicas más que conozcan, escribiendo en su cuaderno las diferentes acepciones de ambas. Después las compartirán en el aula con el resto de sus compañeros y observarán las coincidencias.

Recordaremos a los alumnos el personaje de Mary Poppins. Mary Poppins poseía un bolso inagotable del que siempre podían salir cosas sorprendentes, como el bolso que describe el poema. Les pediremos que hagan una pequeña búsqueda para recordar quién era y cómo era su bolso y expongan oralmente esta información a sus compañeros.

El último verso del poema, "Que va a dar al mar", es una cita que homenajea un famoso verso de nuestra tradición poética en lengua española. Se pedirá a los alumnos que traten de averiguar cuál.

Una vez dilucidado el autor del verso anterior, Jorge Manrique en las célebres $C_{o}$ plas por la muerte de su padre, se pedirá a los alumnos que investiguen sobre este poeta del siglo XV y traigan a clase toda la información encontrada sobre él y su obra literaria, para compartirla con sus compañeros.

Se pedirá a los alumnos que busquen en Internet las Coplas por la muerte de su padre, eligiendo la que más les guste. La compartirán con sus compañeros y escucharán atentamente las escogidas por ellos a su vez.

Reflexionaremos sobre la analogía que Juana Castro traza entre su bolso y "nuestras vidas que son los ríos que van a dar a la mar", como cantara Jorge Manrique. Los alumnos debatirán sobre cuál puede ser el significado interno de este guiño poético, exponiendo las razones en las que se basan para defender su opinión.

Para finalizar, se les propondrá una actividad optativa. Si les ha gustado "Toda la piel del mundo", pueden buscar más información sobre Cartas de enero, el libro de Juana Castro al que pertenece el poema, y compartirla en clase con sus compañeros. Pueden contarles en qué año se publicó, qué ha dicho la crítica de él, de cuántas partes se compone, etc. Pueden también seleccionar otro poema del libro que les guste y traerlo a clase para leerlo en voz alta. 


\section{Criterios de evaluación}

El tratamiento especial que merece la poesía en el aula requiere que su aplicación didáctica no sea evaluable del mismo modo que el resto de contenidos gramaticales o léxicos. De ser así, perdería gran parte del atractivo que posee para el alumno. Hay que recordar que la meta final no es aprender de memoria la biografía de un autor ni una lista de vocabulario, sino interpretar todos los posibles sentidos que tiene el lenguaje utilizado artísticamente y abrir la mente al mundo cultural que subyace bajo las palabras del idioma en curso de aprendizaje.

Los criterios de evaluación del nivel avanzado de español como lengua extranjera incluyen las competencias propias del nivel para cada una de las cuatro destrezas: comprensión oral, comprensión escrita, expresión e interacción oral y expresión e interacción escrita.

Sin embargo, debemos tener siempre presente que la inclusión de la poesía en el aula no es una propuesta didáctica gratuita, sino muy enriquecedora. Y precisamente a causa de su especial carácter de delectación estética, consideramos que sería contraproducente proceder con el mismo esquema evaluativo aplicable al resto de unidades didácticas del curso.

De este modo, los criterios de evaluación particulares para esta propuesta didáctica serían independientes y estarían únicamente basados en el poema seleccionado y en las actividades realizadas. Como ejemplo, los siguientes ítems pueden ser utilizados por el profesor para medir el rendimiento y la implicación de los alumnos en la propuesta didáctica de aplicación de la poesía al aula, puntuando cada uno de ellos como muy logrado, satisfactorio o no logrado:

I. ¿El estudiante muestra interés hacia la poesía española a través de Juana Castro?

2. ¿Reconoce las características y los rasgos identificativos de la poesía de Juana Castro?

3. ¿Mejora su comprensión lectora y su apreciación literaria con el desarrollo de la propuesta?

4. ¿Se observa un aprendizaje progresivo de las distintas habilidades comunicativas?

5. ¿Es capaz de llegar a una comprensión profunda del poema elegido?

6. ¿Logra entender el significado de las palabras por su contexto?

7. ¿Mediante la lectura comprende el texto planteado en clase y es capaz de ver la intertextualidad que de él se desprende?

8. ¿Participa activamente en los debates suscitados con la aplicación didáctica del poema?

9. ¿Es capaz de expresarse con propiedad utilizando todo lo aprendido?

IO. ¿Se observa una actitud positiva hacia la potenciación de la creatividad en el aula? 
II. ¿Es capaz de valorar los contenidos culturales y las propuestas estéticas contenidas en la poesía de Juana Castro?

El presente cuestionario de evaluación es para uso interno del profesor, que podrá ampliarlo o modificarlo según las necesidades específicas de su grupo-clase. Es aconsejable rellenarlo a la finalización de la propuesta didáctica, tras el desarrollo del poema, para de este modo ser capaz de analizar mejor el rendimiento de cada uno de los alumnos.

Por tanto, se trata de una acción didáctica de carácter específico, en la cual no todos los contenidos han de ser evaluables, sino más bien ejercicios instrumentalizados, un incentivo extra para que el estudiante se sumerja en la cultura de la lengua objeto de aprendizaje, así como en su musicalidad y su potencialidad estética. Este tratamiento más distendido potenciará sin duda el atractivo que ejerce la poesía en el marco de enseñanzaaprendizaje del español como lengua extranjera.

\section{Procedimientos de evaluación, instrumentos y registros}

Utilizaremos una serie de instrumentos de evaluación y de registro que pasamos a detallar a continuación, no sin antes mencionar algo que para nosotros resulta fundamental, como bien explicita Quiles Cabrera: “el mediador debe fijar los parámetros en función de los objetivos específicos para cada bloque de tareas, siempre guiados a través de unas pautas centradas en el aprender y formuladas desde una concepción formativa y

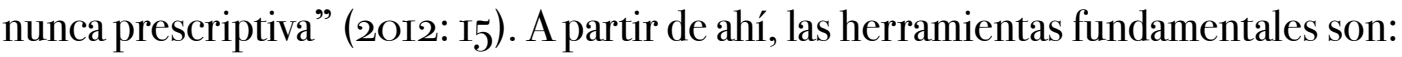

Detección de conocimientos previos sobre el tema que vamos a tratar en el aula. Esta acción nos permite conocer el alcance del conocimiento anterior del alumnado, anticiparnos al grado de interés que suscita y realizar predicciones sobre la temporalización y los resultados.

El Diario de clase supondrá un registro de las diferentes actividades realizadas, las tareas desarrolladas y el grado de aprovechamiento y participación del alumnado.

Cuaderno de notas que refleja los resultados de los ejercicios y de las tareas realizados por los alumnos. Es un instrumento del proceso de evaluación formativa que facilita la posterior implementación del procedimiento de evaluación sumativa.

Actividades de comprensión lectora, escritura creativa y expresión oral susceptibles de ser evaluadas por el profesor, así como el esfuerzo y grado de aprovechamiento personal.

Encuestas de autoevaluación y de coevaluación, que nos ayudarán a detectar el grado de seguimiento de la propuesta didáctica por parte del alumnado. Un ejemplo de rúbrica de autoevaluación o coevaluación podría ser el siguiente:

\section{RÚBRICA DE AUTOEVALUACIÓN Y COEVALUACIÓN DETECCIÓN DEL GRADO DE SEGUIMIENTO DE LA PROPUESTA DIDÁCTICA NIVEL AVANZADO DE LA EEOOII}


ALUMNO/A:

\begin{tabular}{|l|l|l|l|l|}
\hline $\begin{array}{l}\text { Conocimiento } \\
\text { sobre la obra } \\
\text { poética de } \\
\begin{array}{l}\text { Juana Castro } \\
\text { significativamente }\end{array}\end{array}$ & $\begin{array}{l}\text { Ha ha aumentado } \\
\text { aumentado un } \\
\text { poco }\end{array}$ & $\begin{array}{l}\text { Ha aumentado } \\
\text { significativamente }\end{array}$ & $\begin{array}{l}\text { Ha aumentado } \\
\text { significativamente, } \\
\text { con la capacidad } \\
\text { de distinguir } \\
\text { rasgos propios de } \\
\text { su poesia }\end{array}$ \\
\hline $\begin{array}{l}\text { Comprensión } \\
\text { del poema } \\
\text { trabajado }\end{array}$ & No demasiada & $\begin{array}{l}\text { Mejora de la } \\
\text { comprensión, } \\
\text { sin llegar a } \\
\text { abarcar su } \\
\text { globalidad }\end{array}$ & $\begin{array}{l}\text { Comprensión } \\
\text { media sobre el } \\
\text { significado del } \\
\text { poema }\end{array}$ & $\begin{array}{l}\text { La comprensión es } \\
\text { profinda y abraca } \\
\text { aspectos formales } \\
\text { y semánticos }\end{array}$ \\
\hline $\begin{array}{l}\text { Participación } \\
\text { en los debates } \\
\text { orales } \\
\text { suscitados por } \\
\text { el poema }\end{array}$ & No participa & $\begin{array}{l}\text { Escucha } \\
\text { atentamente y } \\
\text { participa } \\
\text { poco }\end{array}$ & $\begin{array}{l}\text { Participa bastante } \\
\text { y aporta puntos de } \\
\text { vista }\end{array}$ & $\begin{array}{l}\text { Participa mucho y } \\
\text { aporta puntos de } \\
\text { vista, relacionando } \\
\text { sus conocimientos } \\
\text { previos con el } \\
\text { poema }\end{array}$ \\
\hline $\begin{array}{l}\text { Actitud hacia } \\
\text { el hecho } \\
\text { poético }\end{array}$ & No mejora & $\begin{array}{l}\text { Mejora leve } \\
\text { de la actitud } \\
\text { personal } \\
\text { hacia el } \\
\text { hecho poético }\end{array}$ & $\begin{array}{l}\text { Mejora sustantiva } \\
\text { de la actitud } \\
\text { personal hacia el } \\
\text { hecho poético }\end{array}$ & $\begin{array}{l}\text { La actitud personal } \\
\text { hacia el hecho } \\
\text { poético se hallaba } \\
\text { muy potenciada de } \\
\text { antemano }\end{array}$ \\
\hline
\end{tabular}

Asimismo, es muy importante disponer de un cuestionario de evaluación propio, que deberá ser respondido una vez que la propuesta haya sido implementada en el aula, con vistas a asegurarnos una mejora de los aspectos cuyo proceso haya resultado menos satisfactorio, así como un ahondamiento en aquellos que más provecho hayan permitido extraer a los alumnos de la aplicación en el aula de la propuesta didáctica.

Algunas de las preguntas del cuestionario podrían ser:

- ¿Qué actividad han disfrutado más de las realizadas en esta propuesta didáctica?

- ¿Con qué parte del poema se han identificado más?

- ¿Son capaces los estudiantes de identificar los rasgos propios de la poesía de Juana Castro?

- ¿Profundizar en la poesía ayuda a los estudiantes a reflexionar sobre ciertos aspectos de la existencia?

- ¿Se han asimilado los conceptos de cohesión, coherencia y adecuación tratados?

- ¿Qué habríamos debido hacer y no hemos hecho?

- ¿Los debates realizados han contribuido a acercar posturas y a fomentar la tolerancia? 
- ¿Las actividades de expresión oral ayudan a la evolución de los aspectos fónicos, gramaticales y léxicos de los estudiantes?

- ¿Han participado activamente los estudiantes en el desarrollo de la propuesta didáctica?

\section{Conclusiones}

Consideramos la poesía como una herramienta pedagógica indispensable en el aula de español como lengua extranjera. Su presencia en la programación didáctica del nivel avanzado de las EEOOII sería muy beneficiosa para el proceso de aprendizaje global del alumnado. Por un lado, se trata de un contacto lingüístico pluridimensional y altamente motivador. Por otro, posee una enorme potencialidad intrínseca para desarrollar la competencia comunicativa de los estudiantes, tanto en su vertiente lingüística como pragmática.

Para el presente trabajo de aplicación didáctica de la poesía de Juana Castro al nivel avanzado del aula de español como lengua extranjera, hemos elegido un poema de esta autora, con el cual hemos construido una propuesta didáctica no evaluable en términos convencionales.

El enfoque comunicativo, predominante en la actualidad en las metodologías de enseñanza de lenguas extranjeras, subraya la importancia del uso de la poesía en el aula, como parte efectiva de la necesaria realia ${ }^{2}$ de la que hay que dotar al alumnado. Para aprovechar todas sus potencialidades, la explotación en el aula debe realizarse tanto desde la vertiente lingüística (gramatical, léxica y fónica) como desde la vertiente pragmática (sociolingüística y cultural). De este modo, la necesaria aplicación didáctica de la poesía al aula podrá ofrecer resultados óptimos en aprendizaje y en motivación.

El poema seleccionado para trabajar con el alumnado de español como lengua extranjera nos ha parecido adecuado desde el punto de vista gramatical, semántico y cultural. Pensamos que acercar la poesía a los estudiantes a través de un autor contemporáneo puede ser una fuente extra de motivación para el aprendizaje de la lengua diana. La poesía, en la enseñanza de español como lengua extranjera, no está suficientemente explotada, a pesar de sus inmensas posibilidades pedagógicas. Nuestra intención es construir un puente entre este género literario y los estudiantes de español, y pensamos que el modo más efectivo de conseguirlo es mediante la implementación en el aula de propuestas didácticas significativas y motivadoras, con un alto potencial de transmisión cultural y valor educativo.

\footnotetext{
${ }^{2}$ El término realia se refiere, en el ámbito de la metodología de enseñanza de lenguas extranjeras, al uso consistente de objetos reales y físicos en el aula, provenientes del mundo exterior. Dicha práctica abarca también los textos auténticos de comunicación lingüística, no diseñados expresamente para el aula. Los poemas son un inmejorable ejemplo de ello, máxime si tenemos en cuenta su vertiente de transmisión cultural y de valores.
} 


\section{Referencias bibliográficas}

- Albaladejo, M. D. (2007). Cómo llevar la literatura al aula de ELE: de la teoría a la práctica. MarcoELE. Revista de Didáctica ELE, 5 , I-5 5 I.

- Campos Fernández-Figares, M. (2004). Planeando la fuga. Puertas a la lectura, I7, (Ejemplar dedicado a: Lecturas y Lenguaje), 46-49.

- Instituto Cervantes (2006). Plan Curricular del Instituto Cervantes. Niveles de referencia para el español, Vol. 3. Madrid: Biblioteca Nueva.

- Jakobson, R. (I963). Essais de linguistique générale. Paris: Minuit.

- Castro, J. (20Io). Cartas de enero, en Heredad seguido de Cartas de enero. Sevilla: Fundación José Manuel Lara.

- Lanseros, R. (20I7). Los poetas toman la palabra. La construcción de la educación literaria en los autores nacidos en posguerra. Madrid: Visor Libros.

- López Valero A. y Encabo Fernández E. (20I3). Fundamentos didácticos de la lengua y la literatura. Madrid: Síntesis.

- Marco común europeo de referencia para las lenguas: aprendizaje, enseñanza y evaluación (20O2). Madrid: MECD.

- Núñez Ruiz, G. (20I4). Lectura literaria y lecturas del mundo: Notas sobre la lectura y la educación literaria. Almería: Universidad de Almería/Red de Universidades Lectoras.

- Quiles Cabrera, M. C. (2OI2). Entre pinceles y libros: textos para un enfoque intertextual e interdisciplinar en el aula de lengua, Álabe, 6, I-I9.

- Real Academia Española (20I4). Diccionario de la lengua española, $23^{\text {a }}$. ed. Madrid: Espasa. 